\title{
Iterative solutions for relativistic dissipative cosmologies
}

\author{
T. Harko and M.K. Mak
}

\begin{abstract}
Iterative solutions of the gravitational field equations for a homogeneous flat Friedmann-Robertson-Walker Universe filled with a causal bulk viscous fluid in the framework of the full Israel-Stewart-Hiscock theory are presented. The general solution of the field equations are presented in a parametric form in the zeroth-, first-, second-, and $m$ th-order approximation by the method of iteration. The time evolution of the scale factor, energy density, deceleration parameter, entropy, Kretschmann scalar, and bulk viscous pressure-thermodynamic pressure ratio for a Zeldovich fluid-filled space-time are discussed.
\end{abstract}

PACS Nos.: 98.80-k, 04.20Jb

Résumé : Dans le cadre de la théorie complète d'Israel-Stewart-Hiscock, nous présentons des solutions itératives pour les équations du champ gravitationnel d'un univers plat homogène de Friedmann-Robertson-Walker dont le volume est rempli d'un fluide visqueux. Nous présentons la solution générale des équations de champ sous forme paramétrique aux ordres d'approximation zéro, un et deux. Nous étudions l'évolution dans le temps du facteur d'échelle, de la densité d'énergie, du paramètre de décélération, de l'entropie, du scalaire de Kretschmann et du rapport entre la pression visqueuse et la pression thermodynamique pour le cas d'un espace-temps rempli d'un fluide de Zeldovich.

[Traduit par la Rédaction]

\section{Introduction}

Dissipative bulk viscous type thermodynamical processes are supposed to play a crucial role in the dynamics and evolution of the early Universe. Over 30 years ago Misner [1] suggested that the observed large-scale isotropy of the Universe is due to the action of the neutrino viscosity that was effective when the Universe was about $1 \mathrm{~s}$ old. There are many processes capable of producing bulk viscous stresses in the early Universe such as interactions between matter and radiation, quark- and gluon-plasma viscosity, and different components of dark matter [2]. Traditionally, for the description of these phenomena the theories of Eckart [3] and Landau and Lifshitz [4] were used (for an extensive review of viscous dissipative cosmologies based on Eckart-type theories see ref. 5). Because of the work in refs. 6-8 it became clear, however, that the Eckart-type theories suffer from serious drawbacks concerning causality and stability. Regardless of the choice of equation of state, all equilibrium states in these theories are unstable, and in addition, signals may be propagated through the fluid at velocities exceeding the speed of light. These problems arise due to the first-order nature of the theory, i.e.,

Received 27 July 2001. Accepted 21 November 2001. Published on the NRC Research Press Web site at http://cjp.nrc.ca/ on 2 May 2002.

T. Harko and M.K. Mak. ${ }^{1}$ Department of Physics, The University of Hong Kong, Pokfulam Road, Hong Kong, P.R. China.

'Corresponding author (e-mail: mkmak@vtc.edu.hk). 
it considers only first-order deviations from the equilibrium. The neglected second-order terms are necessary to prevent noncausal and unstable behavior.

A relativistic second-order theory was found in ref. 6 and developed in ref. 7 into what is called "transient" or "extended" irreversible thermodynamics. However, Hiscock and Lindblom [8] and Hiscock and Salmonson [9] have shown that most versions of the causal second-order theories omit certain divergence terms. The truncated causal thermodynamics of bulk viscosity leads to pathological behavior in the late Universe [9], while the solutions of the full causal theory are well behaved for all times. Therefore, the best currently available theory for analyzing dissipative processes in the Universe is the full Israel-Stewart-Hiscock (ISH) causal thermodynamics. Exact general solutions of the field equations have been obtained very recently in refs. 10-12 for a flat homogeneous Friedmann-Robertson-Walker (FRW) Universe filled with a full causal viscous-fluid source obeying an equation of state for the bulk viscosity coefficient $\xi$ of the form $\xi \sim \rho^{s}$, where $\rho$ is the energy density of the cosmological fluid and $s$ is a constant (for a review of causal thermodynamics and its cosmological and astrophysical applications see ref. 13).

The evolution of the Universe contains a sequence of important dissipative processes, including the GUT (Grand Unified theory) phase transition, taking place at $t \approx 10^{-34} \mathrm{~s}$ and a temperature of about $T \approx 10^{27} \mathrm{~K}$, when gauge bosons acquire mass; reheating of the Universe at the end of inflation $\left(t \approx 10^{-32} \mathrm{~s}\right.$ ), when the scalar field decays into particles; decoupling of neutrinos from the cosmic plasma $\left(t \approx 1 \mathrm{~s}, T \approx 10^{10} \mathrm{~K}\right.$ ), when the temperature falls below the threshold for interactions that keep the neutrinos in thermal contact; nucleosynthesis, decoupling of photons from matter during the recombination era $\left(t \approx 10 \mathrm{~s}, T \approx 10^{3} \mathrm{~K}\right)$, when electrons combine with protons and no longer scatter the photons, etc. [13].

It is the purpose of the present paper to present an iterative scheme for obtaining general solutions of the Einstein gravitational field equations for a causal bulk viscous-fluid-filled flat and homogeneous Universe. By using the Laplace transformation and the convolution theorem, the second-order differential equation describing the evolution of the Hubble parameter $H$ is transformed into an integral equation. Then the general solutions of the equations are obtained in a parametric form in the zeroth-, first-, second- and $m$ th-order approximation by using an iterative method. The approximate solutions of the field equations are compared with an exact solution of the field equations for a dissipative fluid satisfying the stiff equation of state.

The present paper is organized as follows. The physical model and the basic equations are presented in Sect. 2. The method of iterations is applied to the evolution equation in Sect. 3. In Sect. 4, we discuss and conclude our results.

\section{Thermodynamics, field equations, and consequences}

For a flat homogeneous FRW Universe with a line element

$\mathrm{d} s^{2}=\mathrm{d} t^{2}-a^{2}(t)\left(\mathrm{d} x^{2}+\mathrm{d} y^{2}+\mathrm{d} z^{2}\right)$

filled with a bulk viscous cosmological fluid the energy-momentum tensor is given by

$T_{i}^{k}=(\rho+p+\Pi) u_{i} u^{k}-(p+\Pi) \delta_{i}^{k}$

where $\rho$ is the energy density, $p$ the thermodynamic pressure, $\Pi$ the bulk viscous pressure, and $u_{i}$ the four velocity satisfying the condition $u_{i} u^{i}=1$. We use units so that $8 \pi G=c=1$.

The gravitational field equations together with the continuity equation $T_{i ; k}^{k}=0$ imply

$3 H^{2}=\rho, \quad 2 \dot{H}+3 H^{2}=-p-\Pi, \quad \dot{\rho}+3(\rho+p) H=-3 H \Pi$

where $H=\dot{a} / a$ is the Hubble parameter. 
The causal evolution equation for the bulk viscous pressure is given by [14]

$\tau \dot{\Pi}+\Pi=-3 \xi H-\frac{1}{2} \tau \Pi\left(3 H+\frac{\dot{\tau}}{\tau}-\frac{\dot{\xi}}{\xi}-\frac{\dot{T}}{T}\right)$

where $T$ is the temperature, $\xi$ the bulk viscosity coefficient, and $\tau$ the relaxation time. Equation (4) arises as the simplest way (linear in $\Pi$ ) to satisfy the $H$ theorem (i.e., for the entropy production to be non-negative, $S_{; i}^{i}=\Pi^{2} / \xi T \geq 0$, where $S^{i}=e N^{i}-\frac{i \Pi^{2}}{2 \xi T} u^{i}$ is the entropy flow vector, $e$ is the entropy per particle, and $N^{i}=n u^{i}$ is the particle flow vector) $[7,8]$.

To close the system of equations (3,) we have to give the equation of state for $\rho$ and specify $T, \tau$, and $\xi$.

An analysis of the relativistic kinetic equation for some simple cases, given in ref. 15, has shown that in the asymptotic regions of small and large values of the energy density, the viscosity coefficients can be approximated by power functions of the energy density with definite requirements on the exponents of these functions, $\xi \sim \rho^{s}, s=$ const. For small values of the energy density, it is reasonable to consider large exponents, equal in the extreme case to one, $s \approx 1$. For large densities, the power of the bulk viscosity coefficient should be considered smaller (or equal) to $1 / 2, s \leq 1 / 2$.

Therefore, as usual, we shall assume the following phenomenological laws $[14,15]$ :

$p=(\gamma-1) \rho, \quad \xi=\alpha \rho^{s}, \quad T=\beta \rho^{r}, \quad \tau=\xi \rho^{-1}=\alpha \rho^{s-1}$

where $1 \leq \gamma \leq 2$ and $\alpha \geq 0, \beta \geq 0, r \geq 0$, and $s \geq 0$ are constants. Equations (5) are standard in cosmological models whereas the equation for $\tau$ is a simple procedure to ensure that the speed of viscous pulses does not exceed the speed of light.

The requirement that the entropy is a state function imposes in the present model the constraint [10]

$r=\frac{\gamma-1}{\gamma}$

so that $0 \leq r \leq 1 / 2$ for $1 \leq \gamma \leq 2$.

The growth of the total co-moving entropy $\Sigma$ over a proper time interval $\left(t_{0}, t\right)$ is given by [14]

$\Sigma(t)-\Sigma\left(t_{0}\right)=-3 k_{\mathrm{B}}^{-1} \int_{t_{0}}^{t} \Pi H a^{3} T^{-1} \mathrm{~d} t$

where $k_{\mathrm{B}}$ is Boltzmann's constant.

The ISH theory is derived under the assumption that the thermodynamical state of the fluid is close to equilibrium, that is the nonequilibrium bulk viscous pressure should be small when compared to the local equilibrium pressure [16]

$|\Pi|<<p=(\gamma-1) \rho$

If this condition is violated then one is effectively assuming that the linear theory holds also in the nonlinear regime far from equilibrium. For a fluid description of the matter, the condition (8) ought to be satisfied.

To see if a cosmological model inflates or not it is convenient to introduce the deceleration parameter

$q=\frac{\mathrm{d} H^{-1}}{\mathrm{~d} t}-1=\frac{\rho+3 p+3 \Pi}{2 \rho}$

The positive sign of the deceleration parameter corresponds to standard decelerating models whereas the negative sign indicates inflation. 
With these assumptions the evolution equation for flat homogeneous causal bulk viscous cosmological models is [14]

$\ddot{H}+3 H \dot{H}+3^{1-s} \alpha^{-1} H^{2-2 s} \dot{H}-(1+r) H^{-1} \dot{H}^{2}+\frac{9}{4}(\gamma-2) H^{3}+\frac{3^{2-s}}{2 \alpha} \gamma H^{4-2 s}=0$

By introducing a set of nondimensional variables $h$ and $\theta$ by means of the transformations $H=$ $\alpha_{0} h, t=\frac{2}{3 \alpha_{0}} \theta$, with $\alpha_{0}=\left(\frac{3^{s} \alpha}{2}\right)^{\frac{1}{1-2 s}}, s \neq \frac{1}{2}$ and using the expression of $r$ as a function of $\gamma$ given in (6), (10) takes the form

$\frac{\mathrm{d}^{2} h}{\mathrm{~d} \theta^{2}}+\left[2 h+h^{2(1-s)}\right] \frac{\mathrm{d} h}{\mathrm{~d} \theta}-(1+r) h^{-1}\left(\frac{\mathrm{d} h}{\mathrm{~d} \theta}\right)^{2}+\frac{2 r-1}{1-r} h^{3}+\frac{1}{1-r} h^{2(2-s)}=0$

By denoting $n=(1-2 s) /(1-r)$ and changing the variables according to

$h=y^{1 /(1-r)}, \quad \eta=\int y^{1 /(1-r)} \mathrm{d} \theta$

(11) becomes

$\frac{\mathrm{d}^{2} y}{\mathrm{~d} \eta^{2}}+\left(2+y^{n}\right) \frac{\mathrm{d} y}{\mathrm{~d} \eta}+(2 r-1) y+y^{n+1}=0$

\section{Iterative solutions of the evolution equation}

Due to the complicated nonlinear character of the evolution equation (13), very few cosmological solutions of this equation for specific choices of the parameters $r$ and $n$ are known in the framework of the full ISH theory, most of the investigations being devoted to the study of models with $s=1 / 2[10,12]$ or $s=1 / 4$ [19].

The cosmological model presented above, could be robust if the cosmological solutions of (13), depicting the causal bulk viscous FRW space-time, could be studied for an arbitrary range of values of $s$ in the hope of leading to the possibility of correct physical description of a well-determined period in the evolution of our Universe.

By using the Laplace transform and convolution theorem, the differential equation (13) is equivalent with the following integral equation:

$y(\eta)=\int_{0}^{\eta} F(\eta-x)\left[y^{\prime}(x)+y(x)\right] y^{n}(x) \mathrm{d} x+y_{0}(\eta)$

where

$F(\eta-x)=\frac{1}{2 \delta}\left[\mathrm{e}^{-(\delta+1)(\eta-x)}-\mathrm{e}^{(\delta-1)(\eta-x)}\right]$

$y_{0}(\eta)=\mathrm{e}^{-\eta}\left(M \mathrm{e}^{-\delta \eta}+N \mathrm{e}^{\delta \eta}\right)$

$\delta=\sqrt{2(1-r)}$

$M=\frac{(\delta-1) y(0)-y^{*}(0)}{2 \delta}, \quad N=\frac{(\delta+1) y(0)+y^{*}(0)}{2 \delta}$

and we denoted $\left(\frac{\mathrm{d} y}{\mathrm{~d} \eta}\right)_{\eta=0}=y^{*}(0)$.

The solution of the integral equation (14) can be easily obtained by using the method of successive approximations or method of iteration to obtain a solution to any desired accuracy. Taking as an initial 
Fig. 1. Variation of the scale factor $a(t)$ for the stiff causal bulk viscous cosmological fluid for $r=1 / 2$, $s=1 / 4, y(0)=0.5009$, and $y_{0}^{*}=-1.0009$ representing the zeroth- (dotted line), first-, second-, and third-order (longest broken line) approximate solutions.

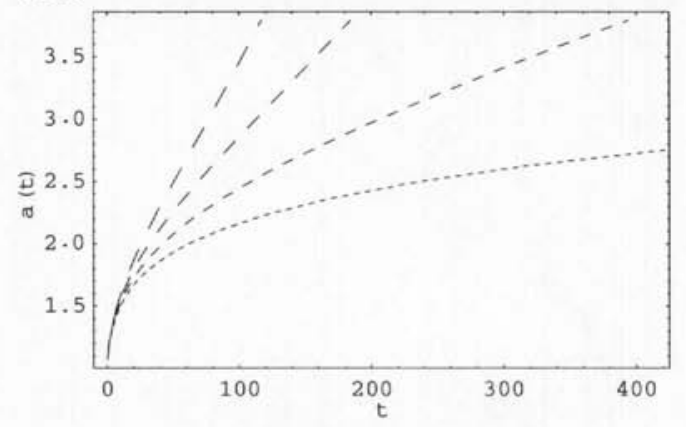

Fig. 2. Variation of the energy density $\rho(t)$ for the stiff causal bulk viscous cosmological fluid for $r=1 / 2$, $s=1 / 4, y(0)=0.5009$, and $y_{0}^{*}=-1.0009$ representing the zeroth- (dotted line), first-, second-, and third-order (longest broken line) approximate solutions.

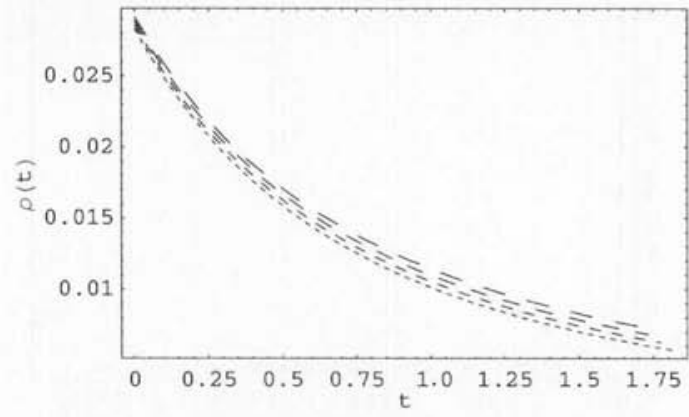

approximation the solution of the linear part of (13), the general solution of the integral equation (14) can be expressed in the first-, second-, and $m$ th-order approximation, $m \in N$, as follows:

$y_{1}(\eta)=\int_{0}^{\eta} F(\eta-x)\left[y_{0}^{\prime}(x)+y_{0}(x)\right] y_{0}^{n}(x) \mathrm{d} x+y_{0}(\eta)$

$y_{2}(\eta)=\int_{0}^{\eta} F(\eta-x)\left[y_{1}^{\prime}(x)+y_{1}(x)\right] y_{1}^{n}(x) \mathrm{d} x+y_{1}(\eta)$

$y_{m-1}(\eta)=\int_{0}^{\eta} F(\eta-x)\left[y_{m-2}^{\prime}(x)+y_{m-2}(x)\right] y_{m-2}^{n}(x) \mathrm{d} x+y_{m-2}(\eta)$

$y_{m}(\eta)=\int_{0}^{\eta} F(\eta-x)\left[y_{m-1}^{\prime}(x)+y_{m-1}(x)\right] y_{m-1}^{n}(x) \mathrm{d} x+y_{m-1}(\eta)$

$y(\eta)=\lim _{m \rightarrow \infty} y_{m}(\eta)$

We can express the iterative solutions of the gravitational field equations for a bulk viscous-fluidfilled FRW Universe in the framework of the full ISH theory in the following parametric form (in the following equations we write $\sigma$ for the variable of integration to distinguish it from the independent variable):

$$
\begin{aligned}
& t-t_{0}=\frac{2}{3 \alpha_{0}} \int_{\eta_{0}}^{\eta} y^{\frac{1}{r-1}}(\sigma) \mathrm{d} \sigma, \quad a=a_{0} \mathrm{e}^{\frac{2}{3} \eta}, \quad \rho=3 \alpha_{0}^{2} y^{\frac{2}{1-r}}(\eta) \\
& p=3(\gamma-1) \alpha_{0}^{2} y^{\frac{2}{1-r}}(\eta), \quad \xi=3^{s} \alpha \alpha_{0}^{2 s} y^{\frac{2 s}{1-r}}(\eta), \quad T=\beta 3^{r} \alpha_{0}^{2 r} y^{\frac{2 r}{1-r}}(\eta) \\
& \tau=\alpha 3^{s-1} \alpha_{0}^{2(s-1)} y^{\frac{2(s-1)}{1-r}}(\eta), \quad q=\frac{3}{2(r-1) y(\eta)} \frac{\mathrm{d} y}{\mathrm{~d} \eta}-1 \\
& \Sigma(t)-\Sigma\left(t_{0}\right)=\frac{6 a_{0}^{3} \alpha_{0}^{2(1-r)}}{k_{\mathrm{B}} \beta 3^{r}} \int_{\eta_{0}}^{\eta}\left[\gamma-\frac{3}{2}(1+q)\right] y^{2}(\sigma) \mathrm{e}^{2 \sigma} \mathrm{d} \sigma
\end{aligned}
$$


Fig. 3. Variation of the deceleration parameter $q(t)$ for the stiff causal bulk viscous cosmological fluid for $r=1 / 2, s=1 / 4, y(0)=0.5009$, and $y_{0}^{*}=-1.0009$ representing the zeroth- (dotted line), first-, second-, and third-order (longest broken line) approximate solutions.

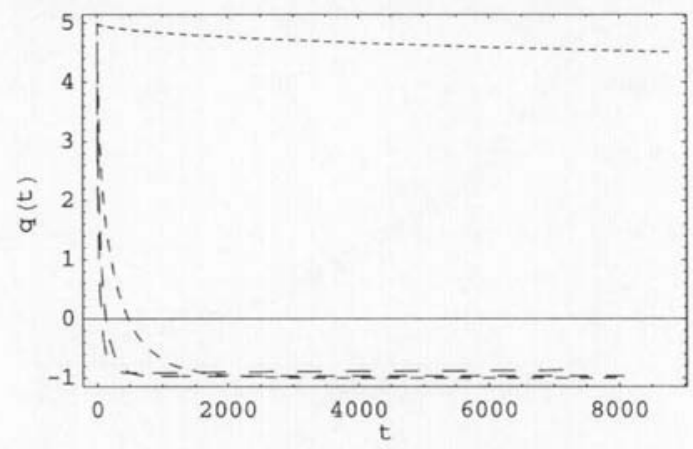

Fig. 4. Variation of the entropy $\Sigma(t)$ for the stiff causal bulk viscous cosmological fluid for $r=1 / 2, s=1 / 4$, $y(0)=0.5009$ and $y_{0}^{*}=-1.0009$ representing the zeroth- (dotted line), first-, second-, and third-order (longest broken line) approximate solutions.

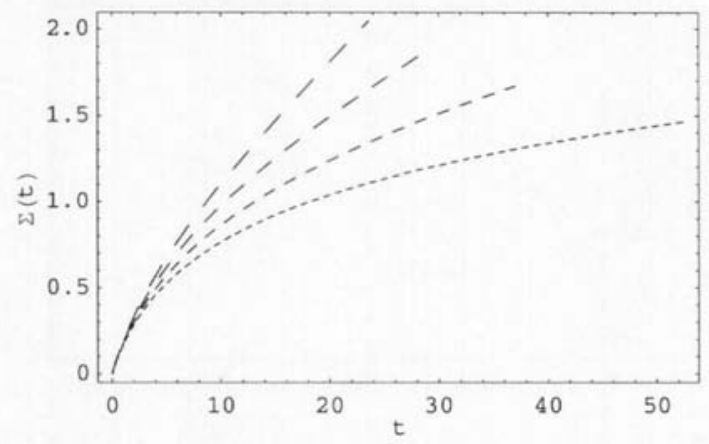

$\Pi=[2(q+1)-3 \gamma] \alpha_{0}^{2} y^{\frac{2}{1-r}}(\eta),|\Pi| p^{-1}=\frac{|2(q+1)-3 \gamma|}{3(\gamma-1)}$

where $a_{0}, t_{0}$, and $\Sigma\left(t_{0}\right)$ are constants of integration.

The nonsingular character of the approximative solutions for all time $t \geq 0$ is consistent with the finite character of the Ricci invariant $R_{i j} R^{i j}$ and Kretschmann scalar $R_{i j k l} R^{i j k l}$

$R_{i j k l} R^{i j k l}=12 \alpha_{0}^{4} y^{\frac{4}{1-r}}(\eta)\left(q^{2}+1\right)=\left(1+\frac{q}{q^{2}-q+1}\right) R_{i j} R^{i j}$

Owing to the form of the dimensionless quantities we have used, this form of the solution of the evolution equation is not defined for $s=1 / 2$.

\section{Discussions and final remarks}

At densities significantly greater than the nuclear one $\rho_{\mathrm{n}}$, e.g., $\rho>>\rho_{\mathrm{n}}$, we have $p \rightarrow \rho$, with the speed of sound, $c_{\mathrm{s}}$, tending to the speed of light, $c_{\mathrm{s}} \rightarrow c$ [18]. A typical approach to the nuclear equation of state in the very high-density regime is to construct a relativistic Lagrangian that allows "bare" nucleons to interact attractively via scalar meson exchange and repulsively via the exchange of a more massive vector meson. But at the highest densities the vector meson exchange dominates and one still has $p=\rho$. Therefore, the equation of state most appropriate to describe the high-density regime of the early Universe is the stiff Zeldovich one, with $\gamma=2$. Hence, a stiff fluid can describe the evolution of the Universe in the GUT era or during the reheating period. In a previous paper [19], we obtained an exact solution for the gravitational field equations of a homogeneous flat FRW Universe filled with a causal bulk viscous fluid satisfying the stiff equation of state $p=\rho$ and having a bulk viscosity coefficient proportional to the fourth root of the energy density $\xi \sim \rho^{1 / 4}$. Hence, it is of interest to study the physical behavior of the approximate solutions for the cases $s=1 / 4$ and $\gamma=2$ for comparison with the exact one. Therefore, for the physical interpretation of the solutions discussed below, we assume the conditions $r=1 / 2$ and $s=1 / 4$.

In the limit of small times, $t \rightarrow 0$, the invariants (29) tend to infinity. Also at this moment, the Universe arrives at a singular state with infinite energy density, bulk viscosity coefficient, and temperature but with a finite scalar factor $a(0)=a_{0}=$ const. The Zeldovich fluid-filled Universe continues to expand and the temperature, energy density, and bulk viscosity coefficient decrease as the cosmic time 
Fig. 5. Variation of the Kretschmann scalar $R_{i j k l} R^{i j k l}$ for the stiff causal bulk viscous cosmological fluid for $r=1 / 2, s=1 / 4, y(0)=0.5009$ and $y_{0}^{*}=-1.0009$ representing the zeroth- (dotted line), first-, second-, and third-order (longest broken line) approximate solutions.

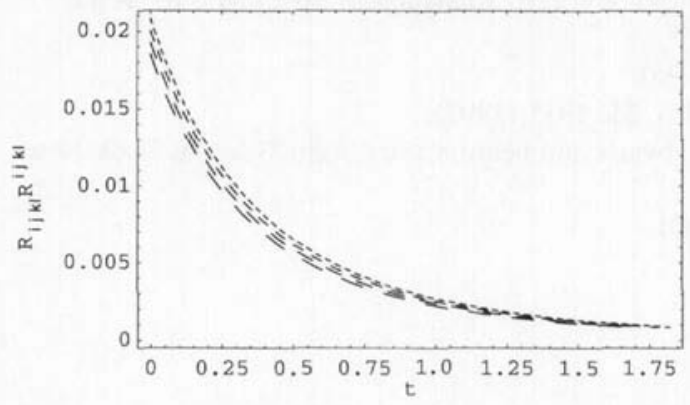

Fig. 6. Variation of the bulk viscous pressurethermodynamic pressure ratio $|\Pi| p^{-1}$ for the stiff causal bulk viscous cosmological fluid for $r=1 / 2$, $s=1 / 4, y(0)=0.5009$ and $y_{0}^{*}=-1.0009$ representing the zeroth- (dotted line), first-, second-, and thirdorder (longest broken line) approximate solutions.

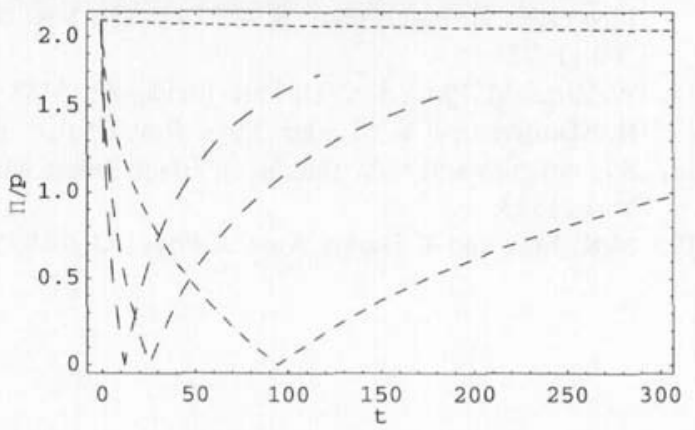

$t$ increases. The evolution of the Universe is noninflationary $(q>0)$ for all times $0 \leq t \leq t_{\mathrm{c}}$, but for times $t>t_{\mathrm{c}}$, the deceleration parameter $q<0$, hence the Universe ends in an inflationary phase.

During the second evolutionary era the expansion is associated with a rapid decrease in the energy density and temperature but a large amount of co-moving entropy is generated. The evolution of the bulk viscous pressure - thermodynamic pressure ratio for all times $0 \leq t \leq t_{\mathrm{p}}$ is less than one showing that the nonequilibrium bulk viscous pressure should be small when compared with the local equilibrium pressure. The bulk viscous pressure is greater than the thermodynamical one, $|\Pi|>p$, for all times $t>t_{\mathrm{p}}$ and consequently, viscous-fluid inflation is a far from equilibrium process, as pointed out by Maartens and Mendez [17].

So, it is a matter of a future analysis and theoretical developments to decide if the inflationary behavior characterizing the present iterative solutions of the gravitational field equations for a FRW Universe filled with a bulk viscous fluid given above, can accurately describe a real physical period in the evolution of our Universe.

In Figs. 1-6, we present the time evolution of the scale factor, energy density, deceleration parameter, entropy, Kretschmann scalar, and bulk viscous pressure - thermodynamic pressure ratio for the Zeldovich fluid-filled FRW Universe representing the zeroth-, first-, second-, and third-order approximation solutions to the Einstein gravitational field equations.

In conclusion, the general physical behavior of this approximate solution describing the Zeldovich fluid-filled FRW Universe presented here is consistent with the exact solutions given in [19].

\section{References}

1. C.W. Misner. Phys. Rev. Lett. 19, 533 (1966).

2. L.P. Chimento and A.S. Jakubi. Phys. Lett. 212A, 320 (1996).

3. C. Eckart. Phys. Rev. 58, 919 (1940).

4. L.D. Landau and E.M. Lifshitz. Fluid mechanics. Butterworth Heinemann, Oxford. 1987.

5. O. Gron. Astrophys. Space Sci. 173, 191 (1990).

6. W. Israel. Ann. Phys. 100, 310 (1976).

7. W. Israel and J.M. Stewart. Phys. Lett. 58A, 213 (1976).

8. W.A. Hiscock and L. Lindblom. Ann. Phys. 151, 466 (1989).

9. W.A. Hiscock and J. Salmonson. Phys. Rev. D: Part. Fields, 43, 3249 (1991).

10. L.P. Chimento and A.S. Jakubi. Class. Quantum Grav. 14, 1811 (1997).

11. L.P. Chimento and A.S. Jakubi. Int. J. Mod. Phys. D, 7, 177 (1998). 
12. M.K. Mak and T. Harko. Gen. Rel. Grav. 30, 1171 (1998); 31, 273 (1999); J. Math. Phys. 39, 5458 (1998); Int. J. Theor. Phys. 38, 1561 (1999); Aust. J. Phys. 52, 659 (1999); Aust. J. Phys. 53, 241 (2000); Int. J. Mod. Phys. D, 9, 97 (2000); 9, 475 (2000).

13. R. Maartens. astro-ph/9609119.

14. R. Maartens. Class. Quantum Grav. 12, 1455 (1995).

15. V.A. Belinskii, E.S. Nikomarov, and I.M. Khalatnikov. Sov. Phys. JETP, 50, 213 (1979); G.L. Murphy. Phys. Rev. D: Part. Fields, 8, 4231 (1973); V.A. Belinskii and I.M. Khalatnikov. Sov. Phys. JETP, 42, 205 (1975).

16. W. Zimdahl. Phys. Rev. D: Part. Fields, 53, 5483 (1996).

17. R. Maartens and V. Mendez. Phys. Rev. D: Part. Fields, 55, 1937 (1997).

18. S.L. Shapiro and S.A. Teukolsky. Black holes, white dwarfs and neutron stars. John Wiley \& Sons, New York. 1983.

19. M.K. Mak and T. Harko. Aust. J. Phys. 53, 241 (2000). 